\title{
Establishment of Survivable Connections in WDM Networks using Partial Path Protection
}

\author{
G. Xue ${ }^{1}$, Senior Member, IEEE, W. Zhang ${ }^{1}$, J. Tang ${ }^{1}$, and K. Thulasiraman ${ }^{2}$, Fellow, IEEE
}

\begin{abstract}
As a generalization of the traditional path protection scheme in WDM networks where a backup path is needed for each active path, the partial path protection scheme uses a collection of backup paths to protect an active path, where each backup path in the collection protects one or more links on the active path such that every link on the active path is protected by one of the backup paths. While there is no known polynomial time algorithm for computing an active path and a corresponding backup path using the path protection scheme for a given source-destination node pair, we show that an active path and a corresponding collection of backup paths using the partial path protection scheme can be computed in polynomial time, whenever they exist, under each of the following two network models: (a) dedicated protection in WDM networks without wavelength converters; and (b) shared protection in WDM networks without wavelength converters. Under each of the two models, we prove that for any given source $s$ and destination $d$ in the network, if one candidate active path connecting $s$ and $d$ is protectable using partial path protection, then any candidate active path connecting $s$ and $d$ is also protectable using partial path protection. This fundamental property leads to efficient shortest active path algorithms that can find an active path and its corresponding partial path protections whenever they exist. Simulation results show that shared partial path protection outperforms shared path protection in terms of blocking probability.
\end{abstract}

Keywords - WDM networks, backup multiplexing, partial path protection, polynomial time algorithms.

\section{INTRODUCTION}

All-optical networks employing wavelength division multiplexing (WDM) and wavelength routing are candidates for future high speed backbone networks [3], [11]. To support mission-critical connection requests, a number of protection schemes for WDM networks have been proposed [1], [4], [7], [8], [9], [13], [15], [18], [19]. Among these schemes, path protection (PP) and link protection (LP) have attracted the most attention [1], [6], [8], [9], [12], [19]. PP is achieved by reserving a backup path which is link-disjoint with the active path so that the traffic on the active path can be rerouted through the backup path when a link along the active path fails. LP is achieved by reserving a backup path for each wavelength channel on the active path. The backup path does not use the link it is protecting. When a link fails, the traffic through a wavelength channel on that link will be rerouted

\footnotetext{
${ }^{1}$ Guoliang Xue, Weiyi Zhang and Jian Tang are all with the Department of Computer Science and Engineering, Arizona State University, Temp, AZ 85287-5406. Email: \{xue, weiyi.zhang, jian.tang\} @ asu.edu. Research supported in part by NSF ITR grant ANI-0312635 and ARO grant W911NF04-1-0385. The information reported here does not reflect the position or the policy of the federal government.

${ }^{2}$ Krishnaiyan Thulasiraman is with the School of Computer Science, University of Oklahoma, Norman, OK 73019. Email: thulasi@ou.edu. Research supported in part by NSF ITR grant ANI-0312435.
}

using its corresponding backup path. A channel on an active path cannot be used by another active path or backup path. In dedicated path/link protection, a channel on a backup path cannot be used by another backup path. In shared path/link protection, a channel on a backup path can be used by another backup path as long as the failure of any link does not activate both backup paths.

In a recent paper [16], Wang, Modiano and Médard introduce the concept of partial path protection (PPP). The idea of PPP is to use a collection of one or more backup paths for each active path, so that the collection of backup paths collectively protect all channels on the active path. They demonstrate that PPP is more powerful than PP in the sense that the existence of PP implies the existence of PPP while the reverse is not true. They consider a dynamic call-bycall system with random arrivals of connection requests and present an ILP formulation to compute an active path and its corresponding PPP with minimum total cost. They also present a shortest active path first (SAPF) heuristic for computing an active path and its corresponding PPP with low total cost. Simulation results demonstrate that the SAPF heuristic has very good performance. Related work can be found in [5], [10], [14], [17].

In this paper, we prove a fundamental property of PPP. In particular, we prove that if partial path protection exists for one candidate active path, then partial path protection exists for any candidate active path. An immediate implication of this property is that we can always use the shortest active path while using PPP. This justifies the use of the SAPF heuristic presented by Wang, Modiano and Médard in [16]. We also present polynomial time algorithms for computing an active path and its corresponding PPP, whenever they exist. Note that computing an active path and its corresponding backup path connecting a source-destination pair using the dedicated path protection scheme in a WDM network without wavelength converters has been shown to be NP-complete by Andersen, Chung, Sen and Xue [2]. More recently, the authors of [13] proved that the problem with shared path protection is also NP-hard. Therefore our polynomial time algorithms demonstrate an important advantage of PPP over PP.

The rest of the paper is organized as follows. In Section II, we present some basic definitions about WDM networks and the protection schemes LP, PP and PPP that will be used in subsequent sections. In Section III, we present a fundamental property of dedicated partial path protection in a WDM network without wavelength converters and a polynomial time algorithm for computing an active lightpath and its dedicated partial path protections, whenever they exist. In 
Section IV, we establish a similar property and an algorithm for shared partial path protection in a WDM network without wavelength converters. In Section $\mathrm{V}$, we present simulation results comparing the performance of partial path protection schemes with their corresponding path protection schemes. We conclude this paper in Section VI.

\section{BASIC DEFINITIONS}

We model a WDM network using an undirected graph $G=$ ( $V, E, \Lambda)$, where $V$ is the set of $n$ vertices, denoting the nodes in the network; $E$ is the set of $m$ edges, denoting the links (or optical fibers) in the network; $\Lambda=\left\{\lambda_{1}, \lambda_{2}, \ldots, \lambda_{W}\right\}$ is the set of $W$ wavelengths each link is capable of carrying. We will use the terms vertices and nodes interchangeably, as well as edges and links. We will use channel to denote a wavelength on a particular link. Specifically, we will use $e^{\lambda}$ to denote the channel which uses wavelength $\lambda$ on link $e$. For any link $e \in E, \Lambda^{A}(e) \subseteq \Lambda$ denotes the set of wavelengths (called active channels) on link $e$ that are used by active paths of existing connections; $\Lambda^{R}(e) \subseteq \Lambda \backslash \Lambda^{A}(e)$ denotes the set of wavelengths (called reserved channels) on link $e$ that are used by backup paths of existing connections; $\Lambda^{F}(e) \subseteq \Lambda \backslash$ $\left\{\Lambda^{A}(e) \cup \Lambda^{R}(e)\right\}$ denotes the set of wavelengths (called free channels) on link $e$ that are not used by either active paths or backup paths of existing connections.

In a WDM network without wavelength converters, data transmission is carried out on a lightpath. Following Chlamtac et. al [3], A lightpath $\pi^{\lambda}(s, d)$ between nodes $s, d \in V$ on wavelength $\lambda \in \Lambda$ is an $s-d$ path $\pi(s, d)$ in $G$ which uses wavelength $\lambda$ on every link of path $\pi(s, d) . \pi(s, d)$ is called the basepath of lightpath $\pi^{\lambda}(s, d)$. $\lambda$ is called the wavelength of lightpath $\pi^{\lambda}(s, d)$. Note that all channels on a lightpath must be on the same wavelength. This is known as the wavelength continuity constraint.

To protect a mission-critical connection from any single link failure, we need to set up an active path and its corresponding backup to protect against the failure of a link along the active path. It is well-known that the backup path should not use any of the links it is protecting. This constraint is enforced in all three commonly known protection schemes: LP, PP, and PPP. Both LP and PP are well studied we refer the readers to [12] for definitions and further reading on LP and PP.

In PPP [16], for every connection request $\rho$ with source node $s(\rho)$ and destination node $d(\rho)$, we need to establish an active path $\mathcal{A P}(\rho)$ connecting $s(\rho)$ and $d(\rho)$. We also need to establish a collection of one or more backup paths $\mathcal{B P}(\rho)$ each connecting $s(\rho)$ and $d(\rho)$ such that for every link $e$ on $\mathcal{A P}(\rho)$, there is a corresponding backup path $\mathcal{B P}(\rho, e) \in \mathcal{B P}(\rho)$ which does not use link $e$, but may share links and/or channels with the rest of $\mathcal{A P}(\rho)$. Note that we may have $\mathcal{B P}\left(\rho, e_{1}\right)=$ $\mathcal{B P}\left(\rho, e_{1}\right)$ for two different links $e_{1}$ and $e_{2}$ on $\mathcal{A P}(\rho)$. Note also that we are talking about a backup path for a channel on the active path. Partial path protection is different from link protection where the backup path for a link connects the two end nodes of the protected link, rather than $s(\rho)$ and $d(\rho)$. Partial path protection is also different from path protection where the backup path protects the entire active path, rather than part of the active path. Again, partial path protection could be either shared or dedicated. In shared partial path protection, the backup path $\mathcal{B P}(\rho, e)$ of one active path $\mathcal{A P}(\rho)$ may share a channel with the backup path $\mathcal{B P}(\sigma, f)$ of another active path $\mathcal{A P}(\sigma)$ if and only if the links on $\mathcal{A P}(\rho)$ that $\mathcal{B P}(\rho, e)$ is supposed to protect do not intersect the links on $\mathcal{A P}(\sigma)$ that $\mathcal{B P}(\sigma, f)$ is supposed to protect. In dedicated partial path protection, the backup path $\mathcal{B P}(\rho, e)$ of one active path $\mathcal{A P}(\rho)$ cannot share a channel with the backup path $\mathcal{B P}(\sigma, f)$ of another active path $\mathcal{A} \mathcal{P}(\sigma)$. However, two backup paths for the same active path may share channels. Fig. 1 illustrates both shared and dedicated partial path protections. Fig. 1(a)

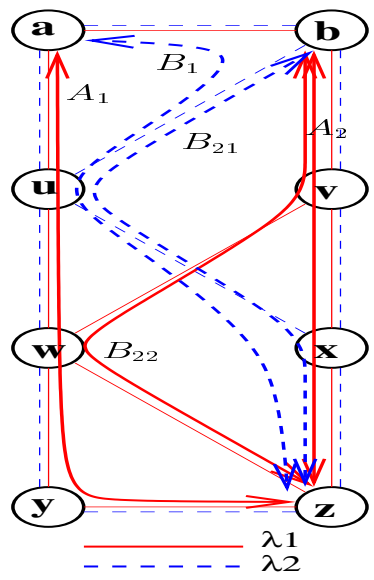

(a)

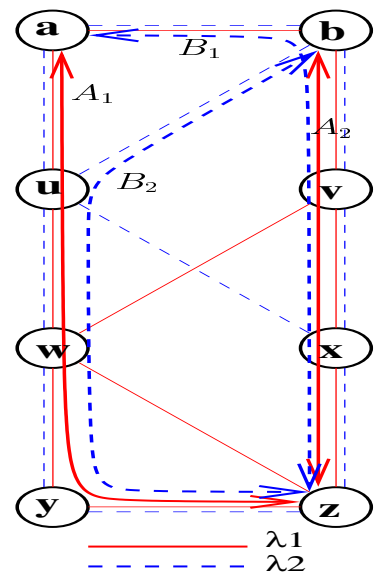

(b)
Fig. 1. (a) Shared partial path protection: $A_{1}(a-u-w-y-z)$ is an active path on $\lambda_{1}, A_{2}(b-v-x-z)$ is an active path on $\lambda_{1}, B_{1}\left(a-b-u-x-z\right.$ on $\left.\lambda_{2}\right)$ is the backup path for all links on $A 1, B_{21}\left(b-u-x-z\right.$ on $\left.\lambda_{2}\right)$ is the backup path for links $b-v$ and $v-x$ on $A_{2}, B_{22}\left(b-v-w-z\right.$ on $\left.\lambda_{1}\right)$ is the backup path for link $x-z$ on $A_{2}$. (b) Dedicated partial path protection: $A_{1}$ is an active path on $\lambda_{1}$, $A_{2}$ is an active path on $\lambda_{1}, B_{1}$ is the backup path for all links on $A_{1}, B_{2}$ is the backup path for all links on $A_{2}$.

illustrates two active paths $A_{1}$ and $A_{2}$ and their corresponding (shared) partial path protections. For $A_{1}$, a single path $B_{1}$ protects all links on $A_{1}$. For $A_{2}$, we have two backup paths. $B_{21}$ is used to protect links $b-v$ and $v-x$ on $A_{2}, B_{22}$ is used to protect link $x-z$ on $A_{2}$. We note that $B_{1}$ and $B_{21}$ share several channels. We also note that $B_{22}$ shares channel $b$ - $v$ (on $\lambda_{1}$ ) with active path $A_{2}$. When link $x-z$ fails, the traffic between $v$ and $z$ on $A_{1}$ will be rerouted via the path $v-w-z$ on $\lambda_{1}$.

Fig. 1(b) illustrates two active paths $A_{1}$ and $A_{2}$ and their corresponding (dedicated) partial path protections. For $A_{1}$, a single path $B_{1}$ protects all links on $A_{1}$. For $A_{2}$, a single path $B_{2}$ protects all links on $A_{2}$. Note that if we fix the protection for $A_{1}$ as in Fig. 1(a), we would not be able to find dedicated partial path protections for $A_{2}$.

Wang, Modiano and Médard [16] have shown that for any given connection request, the existence of an active path and its corresponding path protection implies the existence of an active path and its corresponding partial path protection, but the reverse is not true. Therefore partial path protection is a very promising protection scheme. In the following, we formally define partial path protection under two different 
network models. As in [16], we consider a dynamic callby-call system where connection requests arrive sequentially. For each connection request, we will block it only if it is impossible to establish an active path and its corresponding partial path protections.

Let $e \in E$ be a link in the network. We use $\mathcal{A C}(e)$ to denote the set of connections whose active lightpaths pass through link $e$. We use $\mathcal{B C}(e)$ to denote the set of connections whose backup lightpaths pass through link $e$. We will use existing active path to mean an active path of an existing connection. We will use existing backup path means the backup path for some links on an existing active path. We will use the term active path to mean a candidate for the active of the connection request under consideration.

Let $\rho$ be a connection request with source $s(\rho)$ and destination $d(\rho)$. The lightpath connection with dedicated partial path protection (LPDPPP) problem asks for a lightpath connection between $s(\rho)$ and $d(\rho)$ with dedicated partial path protection. The lightpath connection with shared partial path protection (LPSPPP) problem asks for a lightpath connection between $s(\rho)$ and $d(\rho)$ with shared partial path protection. We will define and address these two problems in the next two sections.

\section{Dedicated Partial Path Protection}

In this section, we concentrate on dedicated partial path protection in a WDM network without wavelength converters.

Definition 1: [Lightpath Connection with Dedicated Partial Path Protection (LPDPPP)] Let $\rho$ be a connection request with source $s(\rho)$ and destination $d(\rho)$. A lightpath connection with dedicated partial path protection for $\rho$ consists of an active path $\mathcal{A L}(\rho)$ and a set of backup paths $\mathcal{B L}(\rho)$ corresponding to $\mathcal{A L}(\rho)$, where $\mathcal{A L}(\rho)$ is a lightpath connecting $s(\rho)$ and $d(\rho), \mathcal{B L}(\rho)$ is a set of lightpaths each connecting $s(\rho)$ and $d(\rho)$ such that the following conditions are satisfied:

A1: The lightpath $\mathcal{A L}(\rho)$ uses free wavelength channels only.

A2: For each link $e$ on $\mathcal{A L}(\rho)$, there is a corresponding lightpath $\mathcal{B L}(\rho, e) \in \mathcal{B L}(\rho)$ such that $\mathcal{B L}(\rho, e)$ does not use link $e$. $\mathcal{B L}(\rho, e)$ is the backup path of link $e$ on $\mathcal{A L}(\rho)$. $\mathcal{B L}(\rho, e)$ may share channels with $\mathcal{A L}(\rho)$. Also, $\mathcal{B L}\left(\rho, e_{1}\right)$ may share channels with $\mathcal{B L}\left(\rho, e_{2}\right)$ for two different links $e_{1}$ and $e_{2}$ on $\mathcal{A}(\rho)$.

A3: Every lightpath in $\mathcal{B} \mathcal{L}(\rho, e)$ uses only free wavelength channels.

Let $\mathcal{A L}(\rho)$ be an $s(\rho)-d(\rho)$ lightpath using only free wavelength channels. We say that lightpath $\mathcal{A L}(\rho)$ is dedicated partial path protectable if there exists a set of backup paths $\mathcal{B L}(\rho)$ such that conditions $\mathrm{A} 1-\mathrm{A} 3$ are satisfied. In this case, we say that $\mathcal{B} \mathcal{L}(\rho)$ is the dedicated partial path protection of active lightpath $\mathcal{A L}(\rho)$.

One can immediately notice the following difference between the traditional path protection scheme and the partial path protection scheme. In path protection, a single backup path is used to protect all links on the corresponding active path. In partial path protection, all links on the active path are protected, but two different links on the active path may be protected using two different backup paths.
A more important, but less obvious, difference between path protection and partial path protection is the following. Let $\rho$ be a connection request specified by a source node $s(\rho)$ and a destination node $d(\rho)$. Computing a pair of link-disjoint lightpaths connecting $s(\rho)$ and $d(\rho)$ is an NP-hard problem, as has been shown by Andersen, Chung, Sen and Xue [2]. However, an active lightpath for $\rho$ and a corresponding dedicated partial path protection can be computed efficiently, as will be shown in this section. In other words, establishing lightpath connection with dedicated path protection is an NP-hard problem while establishing lightpath connection with dedicated partial path protection is polynomial time solvable. In the next three sections, we will show that similar results also hold for the other three network models.

Given a candidate active lightpath connecting the source node and the destination node, the existence of a link-disjoint backup lightpath can be decided efficiently. However, it may happen that for one candidate active lightpath there is a linkdisjoint backup lightpath, but for another candidate active lightpath there is no link-disjoint backup lightpath.

In the following, we will show if one active lightpath connecting a given source-destination node pair is dedicated partial path protectable, then any active lightpath connecting the same source-destination node pair is also dedicated partial path protectable. We will then use this fundamental property to design an efficient algorithm for establishing a lightpath connection with dedicated partial path protection. This fact makes the partial path protection scheme more attractive than the traditional path protection scheme.

Theorem 1: Let $\rho$ be a connection request with source $s(\rho)$ and destination $d(\rho)$. Let $\mathcal{A} \mathcal{L}_{1}(\rho)$ and $\mathcal{A L}_{2}(\rho)$ be two $s(\rho)-d(\rho)$ lightpaths using only free wavelength channels. If there exists a set of lightpaths $\mathcal{B L}_{1}(\rho)$ so that $\mathcal{A L}_{1}(\rho)$ and $\mathcal{B L}_{1}(\rho)$ form a lightpath connection with dedicated partial path connection for $\rho$ with $\mathcal{A L}_{1}(\rho)$ as the active path, then there exists a set of lightpaths $\mathcal{B} \mathcal{L}_{2}(\rho)$ so that $\mathcal{A} \mathcal{L}_{2}(\rho)$ and $\mathcal{B L}_{2}(\rho)$ form a lightpath connection with dedicated partial path connection for $\rho$ with $\mathcal{A} \mathcal{L}_{2}(\rho)$ as the active path. In other words, $\mathcal{A L}_{1}(\rho)$ is dedicated partial path protectable if and only if $\mathcal{A L}_{2}(\rho)$ is dedicated partial path protectable.

PRoOF. We will define $\mathcal{B} \mathcal{L}_{2}(\rho)$ to be the set $\left\{\mathcal{B L}_{2}(\rho, e) \mid e \in\right.$ $\left.\mathcal{A L}_{2}(\rho)\right\}$ with $\mathcal{B L}_{2}(\rho, e)$ defined in the following.

Let $e$ be any link on $\mathcal{A L}_{2}(\rho)$. If $e$ in not on $\mathcal{A L}_{1}(\rho)$, we define $\mathcal{B L}_{2}(\rho, e)=\mathcal{A} \mathcal{L}_{1}(\rho)$. If $e$ is on $\mathcal{A L}_{1}(\rho)$, we define $\mathcal{B L}_{2}(\rho, e)=\mathcal{B L}_{1}(\rho, e)$. We need to show that $\mathcal{A L}_{2}(\rho)$ and $\mathcal{B L}_{2}(\rho)$ satisfy conditions $\mathrm{A} 1-\mathrm{A} 3$ in Definition 1 , i.e., $\mathcal{B} \mathcal{L}_{2}(\rho)$ is a dedicated partial path protection for $\mathcal{A L}_{2}(\rho)$.

Since $\mathcal{A L}_{2}(\rho)$ uses only free wavelength channels by assumption, A1 is satisfied.

For any link $e$ on $\mathcal{A L}_{2}(\rho), \mathcal{B L}_{2}(\rho, e)$ is either $\mathcal{A L}_{1}(\rho)$ (when $e$ is not on $\mathcal{A L}_{1}(\rho)$ ) or $\mathcal{B L}_{1}(\rho, e)$ (when $e$ is on $\mathcal{A L}_{1}(\rho)$ ). Since $\mathcal{B} \mathcal{L}_{1}(\rho, e)$ is the backup path for link $e$ when $e$ is on $\mathcal{A L}_{1}(\rho)$, condition $\mathrm{A} 2$ is satisfied.

When $\mathcal{B L}_{2}(\rho, e)$ is $\mathcal{A L}_{1}(\rho)$, it uses only free channels. When $\mathcal{B L}_{2}(\rho, e)$ is $\mathcal{B L}_{1}(\rho, e)$, it uses only free channels since $\mathcal{B L}_{2}(\rho)$ form a dedicated partial path protection for $\mathcal{A L}_{1}(\rho)$. Therefore condition A3 is satisfied. 
Theorem 1 says that we can use any candidate active lightpath for the current connection request, without affecting the existence of dedicated partial path protection for the active path. As a result, we can always choose to use the shortest active lightpath, leading to an efficient algorithm for establishing a lightpath connection with shared partial path protection listed as Algorithm 1.

\begin{tabular}{ll}
\hline Algorithm 1 LPDPPP \\
\hline INPUT: & Network $G(V, E, \Lambda)$ with known $\mathcal{A C}(e)$ and \\
& $\mathcal{B C}(e)$ for each link $e \in E$; A connection request \\
& $\rho$ with source $s(\rho)$ and destination $d(\rho)$. \\
OUTPUT: & Either block the request or establish an active \\
& lightpath $\mathcal{A} \mathcal{L}(\rho)$ and its dedicated partial path \\
& protections $\mathcal{B} \mathcal{L}(\rho)$.
\end{tabular}

step_1 1 Find shortest active path $\mathcal{A L}(\rho)\}$

Find a minimum hop $s(\rho)-d(\rho)$ lightpath $\mathcal{A L}(\rho)$ using free wavelength channels only.

if $\mathcal{A L}(\rho)$ cannot be found then

stop, block the request.

else

goto the next step, still treating the channels on $\mathcal{A L}(\rho)$ as free.

endif

step_2 $\{$ Find dedicated PPP $\mathcal{B L}(\rho)\}$

Set $\mathcal{B L}(\rho)=\emptyset$.

for each link $e \in \mathcal{A L}(\rho)$ do

Set $G^{\prime}$ to a copy of $G$ and make the following modifications on $G^{\prime}$ :

Set the cost of each free channel not on $\mathcal{A L}(\rho)$ to 1 . Set the cost of each channel on $\mathcal{A L}(\rho)$ or a backup path in $\mathcal{B} \mathcal{L}(\rho)$ to 0 .

Remove all channels on link $e$ and all active channels and reserved channels.

Find a minimum cost $s(\rho)-d(\rho)$ lightpath

$\mathcal{B L}(\rho, e)$ in $G^{\prime}$.

if such a path does not exist then

stop, block the request.

elseif $\mathcal{B} \mathcal{L}(\rho, e) \notin \mathcal{B L}(\rho)$ then

endif

$\mathcal{B} \mathcal{L}(\rho)=\mathcal{B} \mathcal{L}(\rho) \cup\{\mathcal{B} \mathcal{L}(\rho, e)\}$

endfor

step_3 $\{$ Making reservations $\}$

for each channel $e^{\lambda}$ on $\mathcal{A L}(\rho)$ do

mark the channel $e^{\lambda}$ as active.

$\mathcal{A C}(e)=\mathcal{A C}(e) \cup\{\rho\}$.

for each channel $f^{\sigma} \in \mathcal{B L}(\rho, e), f^{\sigma} \notin \mathcal{A L}(\rho)$

mark $f^{\sigma}$ as reserved.

$\mathcal{B C}(f)=\mathcal{B C}(f) \cup\{\rho\}$

endfor

endfor

output $\mathcal{A L}(\rho)$ and $\mathcal{B L}(\rho)$ as the active lightpath and its dedicated partial path protections.

Theorem 2: The worst-case time complexity of Algorithm 1 is $O\left(n^{2} W+n m W\right)$. If a lightpath connection with dedicated partial path protection exists, the algorithm finds an active lightpath $\mathcal{A} \mathcal{L}(\rho)$ and its dedicated partial path protection $\mathcal{B L}(\rho)$; otherwise, the algorithm indicates that the request should be blocked.

PROOF. It follows from Theorem 1 that if there exists a lightpath connection with dedicated partial path protection then any candidate active lightpath is dedicated partial path protectable. Therefore we use the shortest lightpath on free wavelength channels as the candidate active path. If such a lightpath cannot be found, a lightpath connection with dedicated partial path protection does not exist.

Once the candidate active lightpath $\mathcal{A L}(\rho)$ is found, the algorithm tries to find a low cost (measured by the number of free channels to be used) backup path for each channel on $\mathcal{A L}(\rho)$. Again it follows from Theorem 1 that $\mathcal{B L}(\rho)$ can be computed if and only if it exists. This proves the correctness of the algorithm.

To analyze the time complexity, we note that step_1 requires $O(m W+n W \log (n W))$ time. step_2 loops $O(n)$ times, each time taking $O(m W+n W \log (n W))$ time. Therefore the time complexity of step_2 is $O(n m W+$ $\left.n^{2} W \log \left(n^{2} W\right)\right)=O\left(n m W+n^{2} W \log (n W)\right)$ time. step_3 only requires $O\left(n^{2}\right)$ time. Therefore the worst-case time complexity of Algorithm 1 is $O\left(n m W+n^{2} W \log (n W)\right)$. This completes the proof of the theorem.

\section{Shared Partial Path Protection}

In this section, we concentrate on shared partial path protection in a WDM network without wavelength converters.

Definition 2: [Lightpath Connection with Shared Partial Path Protection (LPSPPP)] Let $\rho$ be a connection request with source $s(\rho)$ and destination $d(\rho)$. A lightpath connection with shared partial path protection for $\rho$ consists of an active path $\mathcal{A L}(\rho)$ and a set of backup paths $\mathcal{B L}(\rho)$ corresponding to $\mathcal{A L}(\rho)$, where $\mathcal{A L}(\rho)$ is a lightpath connecting $s(\rho)$ and $d(\rho), \mathcal{B} \mathcal{L}(\rho)$ is a set of lightpaths each connecting $s(\rho)$ and $d(\rho)$ such that the following conditions are satisfied:

B1: The lightpath $\mathcal{A L}(\rho)$ uses free wavelength channels only.

B2: For each link $e$ on $\mathcal{A L}(\rho)$, there is a corresponding lightpath $\mathcal{B L}(\rho, e) \in \mathcal{B L}(\rho)$ such that $\mathcal{B L}(\rho, e)$ does not use link $e$. $\mathcal{B L}(\rho, e)$ is the backup path of link $e$ on $\mathcal{A L}(\rho)$. $\mathcal{B} \mathcal{L}(\rho, e)$ may share channels with $\mathcal{A L}(\rho)$. Also, $\mathcal{B} \mathcal{L}\left(\rho, e_{1}\right)$ may share channels with $\mathcal{B L}\left(\rho, e_{2}\right)$ for two different links $e_{1}$ and $e_{2}$ on $\mathcal{A} \mathcal{L}(\rho)$.

B3: Every lightpath in $\mathcal{B L}(\rho, e)$ uses either free wavelength channels or reserved wavelength channels.

B4: Let $\mathcal{A} \mathcal{L}(\sigma)$ be the active path of a connection request $\sigma$ that was established earlier and still in use that shares a link $e$ with $\mathcal{A L}(\rho)$ (i.e., $\sigma \in \mathcal{A C}(e)$ ). Then $\mathcal{B L}(\rho, e)$ and $\mathcal{B L}(\sigma, e)$ do not share a channel.

Let $\mathcal{A} \mathcal{L}(\rho)$ be an $s(\rho)-d(\rho)$ lightpath using only free wavelength channels. We say that lightpath $\mathcal{A L}(\rho)$ is shared partial path protectable if there exists a set of backup paths $\mathcal{B L}(\rho)$ such that conditions B1-B4 are satisfied. In this case, we say that $\mathcal{B L}(\rho)$ is the shared partial path protection of active lightpath $\mathcal{A L}(\rho)$.

Similarly to the case in the previous section, we have the following result (algorithm omitted due to space limitation). 
Theorem 3: Let $\rho$ be a connection request with source $s(\rho)$ and destination $d(\rho)$. Let $\mathcal{A L}_{1}(\rho)$ and $\mathcal{A L}_{2}(\rho)$ be two $s(\rho)-$ $d(\rho)$ lightpaths using only free wavelength channels. If lightpath $\mathcal{A L}_{1}(\rho)$ is shared partial path protectable then lightpath $\mathcal{A L}_{2}(\rho)$ is also shared partial path protectable. Moreover, for an active path $\mathcal{A L}(\rho)$, we can decide whether it is shared partial path protectable and compute its partial path protections when it exists, within time complexity $O\left(n^{2} W+n m W\right)$.

\section{Simulation Results}

We use PP to denote the shortest active path first path protection heuristic, which first computes a shortest active path as the candidate active path and then computes shortest backup path which is link-disjoint with the candidate active path. We use PPP to denote the shortest active path first partial path protection algorithms presented in this paper, for each of the network models.

We used three randomly generated topologies for this simulation. Topology 1 has 25 nodes, 69 edges, Topology 2 has 50 nodes, 144 edges and Topology 3 has 100 nodes 294 edges. For each network topology, we tested with 5 wavelengths, 10 wavelengths, and 20 wavelengths respectively. A large number of connection requests were generated. The simulation was started from a zero-loaded network for each of the schemes. For each of the two schemes, whenever a connection cannot be supported, it is dropped. Otherwise, the required resource for that connection is reserved on their corresponding network. These results are presented in Tables I and II.

TABLE I

Lightpath Routing with Shared Protection

\begin{tabular}{|c|c|c|c|c|c|c|}
\hline $\mathrm{N}$ & $\mathrm{E}$ & $\mathrm{W}$ & PP & PPP & tPP & tPPP \\
\hline \hline 100 & 294 & 20 & 1098 & 1237 & 14.086 & 35.570 \\
100 & 294 & 10 & 602 & 683 & 7.172 & 17.833 \\
100 & 294 & 5 & 312 & 340 & 4.153 & 9.794 \\
\hline 50 & 144 & 20 & 686 & 769 & 7.635 & 16.359 \\
50 & 144 & 10 & 368 & 404 & 4.119 & 7.847 \\
50 & 144 & 5 & 187 & 200 & 1.746 & 4.300 \\
\hline 25 & 69 & 20 & 426 & 472 & 4.019 & 7.669 \\
25 & 69 & 10 & 217 & 250 & 2.511 & 3.680 \\
25 & 69 & 5 & 111 & 118 & 1.416 & 1.949 \\
\hline
\end{tabular}

TABLE II

Lightpath Routing with Dedicated Protection

\begin{tabular}{|c|c|c|c|c|c|c|}
\hline $\mathrm{N}$ & $\mathrm{E}$ & $\mathrm{W}$ & $\mathrm{PP}$ & $\mathrm{PPP}$ & tPP & tPPP \\
\hline 100 & 294 & 20 & 678 & 603 & 13.791 & 36.070 \\
100 & 294 & 10 & 365 & 316 & 7.562 & 18.956 \\
100 & 294 & 5 & 185 & 163 & 3.892 & 9.386 \\
\hline 50 & 144 & 20 & 445 & 380 & 8.156 & 16.711 \\
50 & 144 & 10 & 235 & 193 & 4.449 & 8.549 \\
50 & 144 & 5 & 112 & 101 & 2.719 & 4.653 \\
\hline 25 & 69 & 20 & 276 & 243 & 4.473 & 7.531 \\
25 & 69 & 10 & 135 & 114 & 2.164 & 4.386 \\
25 & 69 & 5 & 60 & 58 & 1.159 & 1.897 \\
\hline
\end{tabular}

From the tables, we can see that PPP performs better than PP when backup paths may be shared, but performs worse than PP when backup paths are dedicated. Our simulation results are consistent with that reported in [16]. Since shared protection is more efficient in resource usage than dedicated protection, PPP is a good alternative to PP.

\section{CONCLUSIONS}

In this paper, we have studied survivable routing in WDM networks using partial path protection schemes. Depending on whether protection is shared or dedicated, we have formulated and studied two different problems. These are lightpath connection with dedicated partial path protection (LPDPPP) and lightpath connection with shared partial path protection (LPSPPP). For each of the two problems, we have proved that if a candidate active path has partial path protection then every other candidate active path also has partial path protection. From this, it follows that an active path and its corresponding partial path protection can be computed in polynomial time as long as they exist. Simulation results show that PPP outperforms PP when backup paths may be shared.

\section{REFERENCES}

[1] V. Anand and C. Qiao, Dynamic establishment of protection paths in WDM networks, Part I, IEEE ICCCN'2000, pp. 198-204.

[2] R. Andersen, F. Chung, A. Sen and G. Xue, On disjoint path pairs with wavelength continuity constraint in WDM networks, Infocom'04, pp. 524-535.

[3] I. Chlamtac, A. Ganz, and G. Karmi, Lightpath communications: an approach to high bandwidth optical WAN's, IEEE Trans. on Communications, Vol. 40(1992), pp. 1171-1182.

[4] W.D. Grover and D. Stamatelakis, Cycle-oriented distributed preconfiguration: ring-like speed with mesh-like capacity for self-planning network configuration, ICC'98, pp. 537-543.

[5] K.P. Gummadi, M.J. Pradeep and C.S.R. Murthy, An efficient primarysegmented backup scheme for dependable real-time communication networks, IEEE/ACM Trans. on Networking, Vol. 11(2003), pp. 81-94.

[6] A. Fumagalli, L. Valcarenghi, IP restoration versus WDM protection: is there an optimal choice? IEEE Networks, Vol. 14, No. 6(2000), pp. 3441.

[7] P.H. Ho and H.T. Mouftah, A framework of service guaranteed shared protection for optical networks, IEEE Communications Magazine, Vol. 40, No. 2(2002), pp. 97-103.

[8] M. Médard, S.G. Finn, R.A. Barry and R.G. Gallager, Redundant trees for preplanned recovery in arbitrary vertex-redundant or edge-redundant graphs, IEEE/ACM Trans. on Networking, Vol. 7(1999), pp. 641-652.

[9] M. Médard, R.A. Barry, S.G. Finn, W. He and S.S. Lumetta, Generalized loop-back recovery in optical mesh networks, IEEE/ACM Trans. Networking, Vol. 10(2002), pp. 153-164.

[10] G. Mohan, C.S. Ram Murthy and Arun Somani, Efficient algorithms for routing dependable connections in WDM optical networks, IEEE/ACM Trans. on Networking, Vol. 9(2001), pp. 553-566.

[11] B. Mukherjee, Optical Communication Networks, McGraw Hill, 1997.

[12] S. Ramamurthy and B. Mukherjee, Survivable WDM mesh networks. Part I-Protection Infocom'99, pp. 744-751.

[13] C. Ou; J. Zhang; H. Zang; L.H. Sahasrabuddhe and B. Mukherjee; New and improved approaches for shared-path protection in WDM mesh networks; Journal of Lightwave Technology; Vol. 22(2004), pp. 12231232.

[14] C.V. Saradhi and C.S. Ram Murthy, Segmented protection paths in WDM mesh networks, HPSR'03, pp. 311-316.

[15] D.A. Schupke, W.D. Grover, and M. Clouqueur; Strategies for enhanced dual failure restorability with static or reconfigurable p-cycle networks; IEEE ICC'04, pp. 1628-1633.

[16] H. Wang, E. Modiano and M. Médard, Partial path protection for WDM networks: End-to-end recovery using local failure information, IEEE ISCC'02, pp. 719-725.

[17] D. Xu, Y. Xiong and C. Qiao, Novel algorithms for shared segment protection, IEEE JSAC-OCN, Vol. 21(2003), pp. 1320-1331.

[18] G. Xue, L. Chen and K. Thulasiraman, QoS issues in redundant trees for protection in vertex-redundant or edge- redundant networks, IEEE ICC'02, pp. 2766-2770.

[19] G. Xue, L.Chen and K.Thulasiraman, Quality of service and quality of protection issues in preplanned recovery schemes using redundant trees, IEEE JSAC-OCN, Vol. 21(2003), pp. 1332-1345. 